\title{
Type 3 intestinal failure after bypass grafting for isolated acute superior mesenteric artery dissection: A case report
}

\author{
Ahmed Elshiekh (D), Nick M Matharu(D) \\ Department of Surgery, University Hospitals Coventry and Warwickshire NHS Trust, Coventry, United Kingdom
}

\begin{abstract}
Isolated superior mesenteric artery (SMA) dissection is a very rare cause of mesenteric ischemia. A 66-year-old male patient was admitted to our emergency department with severe epigastric pain. He was diagnosed with spontaneous SMA dissection leading to intestinal ischemia. A bypass graft was used to relieve acute ischemia with an initial success. However, this success was not sustained, as the graft failed slowly despite a satisfactory technical appearance, leading to type 3 intestinal failure which could be explained by the competitive flow. In conclusion, bypass grafting may yield short-term bowel viability in the treatment of isolated SMA dissection and acute ischemia; however, grafts may fail without radiologically apparent technical complications possibly due to the competitive flow from the collateralizing circuits.

Keywords: Intestinal failure, mesenteric bypass, mesenteric ischemia, superior mesenteric artery dissection.
\end{abstract}

Isolated superior mesenteric artery (SMA) dissection was first described in the literature in 1947. ${ }^{[1]}$ Although it is relatively rare, it is the second most common site after the carotid artery for isolated peripheral artery dissection. ${ }^{[1]}$ The widespread application of advanced tools for detection, particularly computed tomography (CT), may explain the recent rise in the number of cases. ${ }^{[2]}$ Interestingly, two-thirds of the reported cases are from the Far East with $86 \%$ in male gender.

The etiology of spontaneous SMA dissection still remains unclear and associated risk factors have not been well identified. Although hypertension was previously put forward as a risk factor, it was only described in $31 \%$ of the reported cases without any pathology other than dissection based on histological examination results. ${ }^{[3]}$ Mechanical stress has been also suggested to be involved in the etiology through examining simulated hemodynamic flow on computer simulation models of CT scans of the diseased arteries. ${ }^{[3,4]}$

Superior mesenteric artery dissection can occur with aortic dissection (AD). It has been reported that $5 \%$ of
$\mathrm{AD}$ cases are associated with intestinal ischemia with an overall higher mortality rate ranging from 36 to $90 \%{ }^{[5,6]}$

According to the literature data, SMA dissection can be classified into three types: the first having a patent true lumen alongside a patent false lumen with re-entry; the second having only a patent true lumen with either a thrombosed or patent false lumen without re-entry; and a third with both lumens thrombosed. ${ }^{[3]}$

Herein, we report a rare case of type 3 intestinal failure after bypass grafting for isolated acute SMA dissection.

\section{CASE REPORT}

A 66-year-old male patient was admitted to our emergency department complaining of severe epigastric pain. His past medical history revealed hypertension controlled by medications. He smoked 10 to 15 cigarettes a day for the last 40 years. He was cold and clammy on examination with signs of peripheral shutdown. Elevated lactate levels $(7.3 \mathrm{mmol} / \mathrm{L})$ and white blood count $\left(14.6 \times 10^{9} / \mathrm{L}\right)$ with abnormal

Received: June 04, 2019 Accepted: June 27, 2019 Published online: July 19, 2019

Correspondence: Ahmed Elshiekh, MD. Department of Surgery, University Hospitals Coventry and Warwickshire NHS Trust, CV2 2DX Coventry, United Kingdom. e-mail: dr.ahmed.elshiekh1@gmail.com 
C-reactive protein values were found in his laboratory studies.

Computed tomography scan showed thrombosis of the proximal portion of the SMA secondary to an extensive dissection flap just after the vessel origin (Figures 1 and 2). The origin of the right colic and ileocolic arteries though patent were shown in the CT scan to arise from the false lumen. The true lumen was thrombosed distal to these origins involving the proximal to mid-small bowel branches.

The significance of this appearance was not initially appreciated, and midline laparotomy was performed by the general surgeons for possible bowel infarction. This revealed generalized dusky-looking small bowel with no evidence of gangrene or perforation. Based on these findings, a vascular surgical opinion was sought. On attendance, review of the scans revealed the above and a more detailed intraoperative inspection showed that the stomach, duodenum, and colon appeared pink and healthy. The SMA at the root of the small bowel mesentery was found to be non-pulsating. The SMA was, then, identified using Doppler ultrasound which demonstrated an occluded flow distal to the origin. Therefore, SMA arteriotomy was performed and cleared proximally with a Size 2 Fogarty catheter. The detached intima was retrieved, but without restoration of the inflow. However, there was good back-bleeding.

The intimal tear was tacked distally within the SMA vessel wall, and a reversed long saphenous vein graft was harvested from the leg and anastomosed from the ipsilateral common iliac artery (CIA) to the SMA bypassing the occluded proximal section. Doppler ultrasound was used to confirm the blood

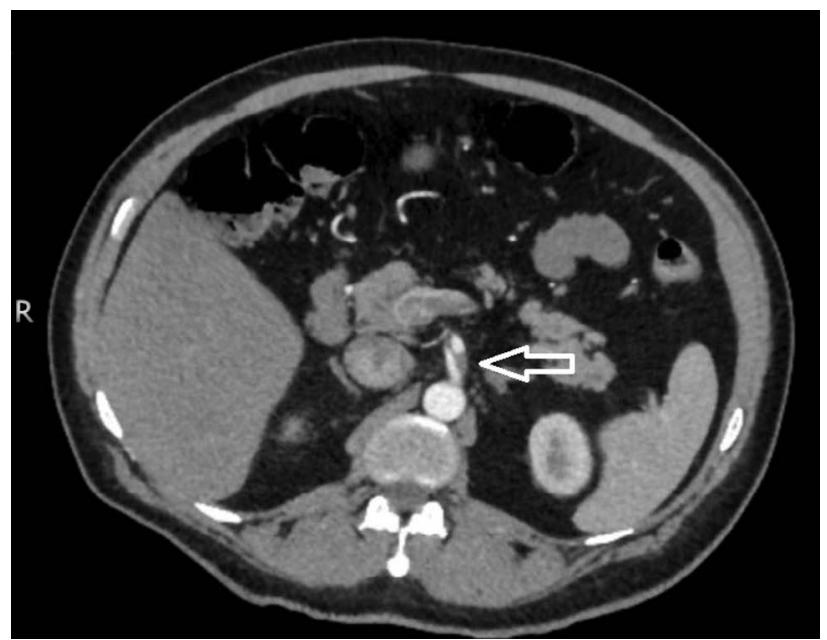

Figure 1. A transverse section on computed tomography scan showing dissection in superior mesenteric artery (arrow). flow which was found to be satisfactory with improved small bowel appearance after the application of warm packs as evidenced by the color and peristalsis.

On postoperative Day 3, the patient suffered from the right iliac fossa tenderness with a sustained elevation of his lactate levels $(2.5$ to $3 \mathrm{mmol} / \mathrm{L})$ and, therefore, another CT scan was performed which showed low flow within a patent vein graft. A better flow to the SMA territories from the collateral routes was demonstrated together with some small patent outflow vessels from the graft. The dissected SMA remained occluded proximally with poor bowel wall enhancement, consistent with ischemia, particularly of small bowel loops in the right side of the abdomen.

Following the CT scan, the Vascular \& Endovascular Surgery Multi-Disciplinary Team (MDT) discussed the case and advised that, although a short section of the SMA was poorly perfused, the SMA proximally and distally to this was well perfused through collateralization from a now widely patent coeliac axis and, thus, the bowel perfusion was no longer dependent on the bypass graft. Therefore, no further endovascular or open vascular surgical interventions were advised, as they would unlikely to improve bowel perfusion. The MDT recommended referral to a specialist gastrointestinal surgical and nutrition team for further management and follow-up.

The patient continued to have significant pain on eating, significant hypoalbuminemia, and temperature spikes, despite antibiotic treatment. Edematous viable

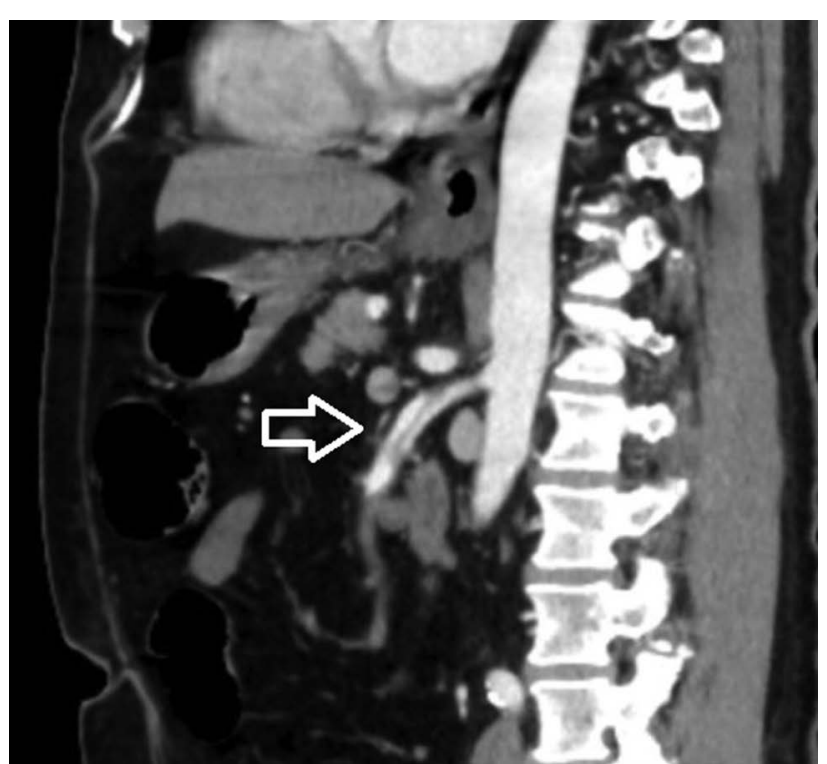

Figure 2. An anteroposterior view on computed tomography scan showing dissection in superior mesenteric artery (arrow). 
bowel and multiple adhesions were found, when a second laparotomy was performed for these symptoms.

Postoperatively, the patient could not tolerate food due to pain, nausea, and loss of appetite and, therefore, colonoscopy was performed which showed no abnormalities apart from slightly granular appearance of the mucosa in the transverse and left colon. The patient was managed conservatively and started nutritional support with total parenteral nutrition (TPN) through a peripherally inserted central line with which he was discharged home.

However, he was readmitted with progressive abdominal pain associated with intermittent vomiting and fever and an episode of postural collapse. He was diagnosed with type 3 intestinal failure and repeated $\mathrm{CT}$ scan showed diffuse thickening and hyperenhancement of the distal small bowel up to the ileocecal junction associated with stranding and small amount of free mesenteric fluid. The ileocolic arteries did not enhance and the bypass graft failed.

Exploratory laparotomy was performed. Most of the ileum was well perfused and had a normal appearance with good peristalsis. However, there was a segment of approximately $50-\mathrm{cm}$ of the distal ileum which was thickened, stiff, and pale with dense adhesions. A bounding pulse was felt in the small bowel mesentery root. This segment was resected and a double-barrelled ileostomy was exteriorized.

The patient recovered well from the operation with no further pain and his appetite improved. At one-year follow-up, full recovery with return to normal body weight preoperatively was achieved. A written informed consent was obtained from the patient.

\section{DISCUSSION}

Isolated SMA dissection is a very rare condition. Several treatment options are available including conservative medical treatment, stenting, bypass, endarterectomy, or coil embolization. Due to its rarity, there is a very limited number of high-quality data in the literature regarding the most optimal treatment.

The main presenting symptom is usually abdominal pain in about $86 \%$ of patients and some studies have shown a direct relationship between the severity of dissection and degree of pain..$^{[4,7-9]}$ The lack of symptoms indicates a higher chance of success of conservative medical treatment. ${ }^{[4]}$ Similarly, our patient also initially presented with abdominal pain.
An algorithm for management has been suggested by Cho et al. ${ }^{[10]}$ and Casella et al. ${ }^{[11]}$ who mainly classified patients as symptomatic and asymptomatic. Conservative management is recommended for asymptomatic patients, while intervention either in the form of surgery or stenting is advised for symptomatic patients or asymptomatic patients unresponsive to conservative treatment. ${ }^{[10,11]}$ Our case was symptomatic with a poor bowel condition and, thus, we opted for intervention.

In the presented case, bypass grafting relieved acute ischemia, but failed gradually after without apparent technical reasons, as collaterals developed. Computed tomography which was performed in the postoperative period showed more flow from the collateral routes than from the vein graft, probably due to the competitive flow. Similar cases have been also described in the literature using crossover grafts for leg ischemia in aortic dissection. ${ }^{[12]}$

The concept of competitive flow can be explained by the fact that the distal segment of the initially diseased vessel now receives blood from two sources: the collateralized blood supply from the coeliac axis and the bypass graft. This was also described before in cardiac surgery. ${ }^{[13-15]}$ This may be due to the unbeneficial shear stress distribution, leading to endothelial dysfunction due to the high competitive flow. ${ }^{[14]}$

With graft occlusion, the flow could be enough to keep the intestine viable, but not to function properly, resulting in multiple nutritional deficiencies. This could be the reason the patient did not tolerate re-feeding, thereby, needing nutritional support in the form of TPN.

In conclusion, bypass grafting can ensure shortterm bowel viability in the treatment of isolated SMA dissection and acute ischemia. However, grafts may fail without radiologically apparent technical complications possibly due to the competitive flow from collateralizing circuits. This results in a dynamically changing perfusion picture which may initially appear adequate, but eventually result in focal chronic bowel ischemia in watershed areas.

\section{Declaration of conflicting interests}

The authors declared no conflicts of interest with respect to the authorship and/or publication of this article.

\section{Funding}

The authors received no financial support for the research and/or authorship of this article. 


\section{REFERENCES}

1. Bauersfeld SR. Dissecting aneurysm of the aorta; a presentation of 15 cases and a review of the recent literature. Ann Intern Med 1947;26:873-89.

2. Okada M, Ishiguchi T, Itoh H. Management of spontaneous dissection of the superior mesenteric artery. Intern Med 2004;43:451-2.

3. Garrett HE Jr. Options for treatment of spontaneous mesenteric artery dissection. J Vasc Surg 2014;59:1433-9.

4. Park YJ, Park CW, Park KB, Roh YN, Kim DI, Kim YW. Inference from clinical and fluid dynamic studies about underlying cause of spontaneous isolated superior mesenteric artery dissection. J Vasc Surg 2011;53:80-6.

5. Slonim SM, Miller DC, Mitchell RS, Semba CP, Razavi MK, Dake MD. Percutaneous balloon fenestration and stenting for life-threatening ischemic complications in patients with acute aortic dissection. J Thorac Cardiovasc Surg 1999;117:1118-26.

6. Fattori R, Cao P, De Rango P, Czerny M, Evangelista $\mathrm{A}$, Nienaber $\mathrm{C}$, et al. Interdisciplinary expert consensus document on management of type B aortic dissection. J Am Coll Cardiol 2013;61:1661-78.

7. Yun WS, Kim YW, Park KB, Cho SK, Do YS, Lee KB, et al. Clinical and angiographic follow-up of spontaneous isolated superior mesenteric artery dissection. Eur J Vasc Endovasc Surg 2009;37:572-7.

8. Picquet J, Abilez O, Pénard J, Jousset Y, Rousselet MC, Enon B. Superficial femoral artery transposition repair for isolated superior mesenteric artery dissection. J Vasc Surg 2005;42:788-91.

9. Watring NJ, Smith CM, Stokes GK, Counselman FL. Spontaneous superior mesenteric artery (SMA) dissection: an unusual cause of abdominal pain. J Emerg Med 2010;3:576-8.

10. Cho BS, Lee MS, Lee MK, Choi YJ, Kim CN, Kang YJ, et al. Treatment guidelines for isolated dissection of the superior mesenteric artery based on follow-up CT findings. Eur J Vasc Endovasc Surg 2011;41:780-5.

11. Casella IB, Bosch MA, Sousa WO Jr. Isolated spontaneous dissection of the superior mesenteric artery treated by percutaneous stent placement: case report. J Vasc Surg 2008;47:197-200.

12. Brief DK, Brener BJ, Alpert J, Parsonnet V. Crossover femorofemoral grafts followed up five years or more. An analysis. Arch Surg 1975;110:1294-9.

13. Nordgaard H, Nordhaug D, Kirkeby-Garstad I, Løvstakken L, Vitale N, Haaverstad R. Different graft flow patterns due to competitive flow or stenosis in the coronary anastomosis assessed by transit-time flowmetry in a porcine model. Eur J Cardiothorac Surg 2009;36:137-42.

14. Ding J, Liu Y, Wang F, Bai F. Impact of competitive flow on hemodynamics in coronary surgery: numerical study of ITA-LAD model. Comput Math Methods Med 2012;2012:356187.

15. Villareal RP, Mathur VS. The string phenomenon: an important cause of internal mammary artery graft failure. Tex Heart Inst J 2000;27:346-9. 